\title{
Amphetamine Alters the Circadian Locomotor Activity Pattern of Adult WKY Female Rats
}

\author{
Cathleen G. Jones, Pamela B. Yang, Victor T. Wilcox, Nachum Dafny* \\ Department of Neurobiology and Anatomy, The University of Texas-Medical School at Houston, Houston, USA \\ Email: $\underline{\text { Nachum.Dafny@uth.tmc.edu }}$
}

Received 26 March 2014; revised 30 April 2014; accepted 8 May 2014

Copyright (C) 2014 by authors and Scientific Research Publishing Inc.

This work is licensed under the Creative Commons Attribution International License (CC BY). http://creativecommons.org/licenses/by/4.0/

(c) (i) Open Access

\begin{abstract}
There are no reports on the effect of amphetamine on female WKY circadian activity pattern. The objective of this study is to investigate whether repeated daily exposure to the psychostimulant amphetamine alters the locomotor circadian rhythm activity patterns of female adult WistarKyoto (WKY) rats. Twenty-four rats were divided into two groups, control $(\mathrm{N}=12)$ and experimental $(\mathrm{N}=12)$, and kept in a 12:12 h light/dark cycle in an open field cage. After 5 to 7 days of acclimation, 11 days of consecutive non-stop recordings began. On experimental day 1 , all groups were given an injection of saline. On experimental days 2 to 7 , the experimental group was injected with $0.6 \mathrm{mg} / \mathrm{kg}$ amphetamine and the control group with saline followed by a washout phase from experimental day 8 to 10, and amphetamine re-challenge or saline on experimental day 11 similar to experimental day 2 . Locomotor movements were determined using a computerized animal activity monitoring system, and cosine statistical analysis was used to fit a24-hour curve to the activity pattern. The horizontal activity (HA), total distance (TD), number of stereotypy (NOS), and stereotypical movements (SM) were analyzed for alterations in the circadian rhythm activity patterns. The data demonstrated that chronic amphetamine administration alters the mesor parameter of the circadian rhythm activity patterns, indicating that chronic amphetamine treatment exerts long term effects on these rats.
\end{abstract}

\section{Keywords}

Acute, Chronic, Amphetamine, Locomotor Activity, Sensitization, Withdrawal

${ }^{*}$ Corresponding author. 


\section{Introduction}

Attention deficit/ hyperactivity disorder (ADHD) is the most common pediatric behavioral disorder in the United States affecting 2 to $18 \%$ of school-aged children [1]. ADHD is characterized by debilitating inattention, hyperactivity, and impulsiveness often associated with other behavioral and learning disorders. The psychostimulants amphetamine and methylphenidate have been the gold standard for treating ADHD for decades. There is consistent evidence that amphetamine enhances sustained attention in normal humans [2] and rats [3] and reduces distractibility [4]. These drugs target the dopamine system leading to an increase in extracellular dopamine (DA) concentration and to a lesser degree they block norepinephrine and serotonin transporters [5]. Although it is still unclear how amphetamine treatment leads to attention improvements, a logical possibility is that the drug acts to modulate sensory processes in the motive circuit altering the threshold for distraction and enhancing sustained attention with reduced distractibility.

All physiological processes of mammals are regulated by about a 24 hour cycle that maintains internal homeostasis and metabolism despite variation in external or internal conditions. Endogenous neurotransmitter, neuromodulator, hormone levels, and behavioral activity exhibit a consistent circadian rhythm activity pattern that is modulated by psychostimulant administration. Alteration of the diurnal activity pattern by drug administration is considered to be an experimental marker for the long term effect of the drug on the animals [6]-[8]. Many drugs, including psychostimulants, show varying pharmacokinetics over the course of a 24 hour day and furthermore elicit behavioral sensitization responses based on the time of treatment, e.g. only when administrated in the morning [9]-[17]. The time of drug exposure is essential to its effect, once the changes occur it was suggested that this change means that the drug exerts long term effects [18] [19]. The administration of psychostimulants such as methylphenidate and cocaine has been shown to elicit either tolerance or sensitization and modulation of the circadian locomotor activity rhythm as well as causing cross sensitization and tolerance dependent on the dose, duration of the treatment, frequency of the treatment, pattern of drug administration, time of drug administration, and environmental factors [9]-[12] [14] [15].

Most of the psychostimulant studies report data obtained from acute exposure of amphetamine on male animal models. The present study uses acute and chronic amphetamine exposure on female animal models. The hypothesis of this study is that chronic amphetamine administration changes the locomotor circadian activity pattern, an experimental indication of the drugs long term effect on circadian clocks that regulate and control diurnal activity.

\section{Materials and Methods}

\subsection{Animals}

Two groups of female Wistar-Kyoto (WKY) rats (total $\mathrm{N}=24$ ), 60-71 days old, were purchased from Charles River (Wilmington, MA) for this experiment. Animals were housed in a sound proof room from their arrival until the end of the experiment. The same cage with corn cob bedding 1/8" (\#CC8) that served as the homecage was also the test cage throughout the experiment. The ambient temperature of the room was $21 \pm 2^{\circ} \mathrm{C}$ with relative humidity of 37\%-42\%. Animals were maintained on a 12:12 h light/dark (06:00-18:00 h light on) with food and water given ad libitum. Animals were kept 5-7 days for acclimation. One day prior to the initial recording, they were randomly divided into two groups, control (saline) and amphetamine $(0.6 \mathrm{mg} / \mathrm{kg}$ i.p.) group (each $\mathrm{N}=$ 12) (see Table 1), and kept for additional 24 hours adaptation prior to the first injection. The duration of the experiment was for eleven non-stop recording days. Every morning for about 20 to30 minutes the recordings were stopped for supplementing food, water and injections. This experimental protocol was adapted from previous dose-response experiments with amphetamine and other psychostimulants [9]-[11] [16] [17].

\subsection{Apparatus}

The locomotor activity home/testing chambers consisted of 16 clear acrylic cages, open-field boxes each 40.5 $\mathrm{cm} \times 40.5 \mathrm{~cm} \times 31.5 \mathrm{~cm}$ (AccuScanInstruments, Inc., Columbus, $\mathrm{OH}$ ) fitted with two arrays of 16 infrared motion sensors located 6 and $12.5 \mathrm{~cm}$ above the floor of the box. This system has been previously described in detail [9]-[12] [14] [18] [19]. In short, the activity monitoring system checked each of the sensor beams at a frequency of $100 \mathrm{~Hz}$ to determine whether beams were interrupted. The interruptions of these beams were compiled by AccuScan Analyzer, and downloaded to a PC every 10 minutes and analyzed using the OASIS program. 
Table 1. Treatment protocol involving adult female rats during the 11 experimental days. 1 to 7 : indicate the comparison between experimental day 1 (the control recording) to experimental day 7 , the last maintenance day of amphetamine administration (see Table 1).

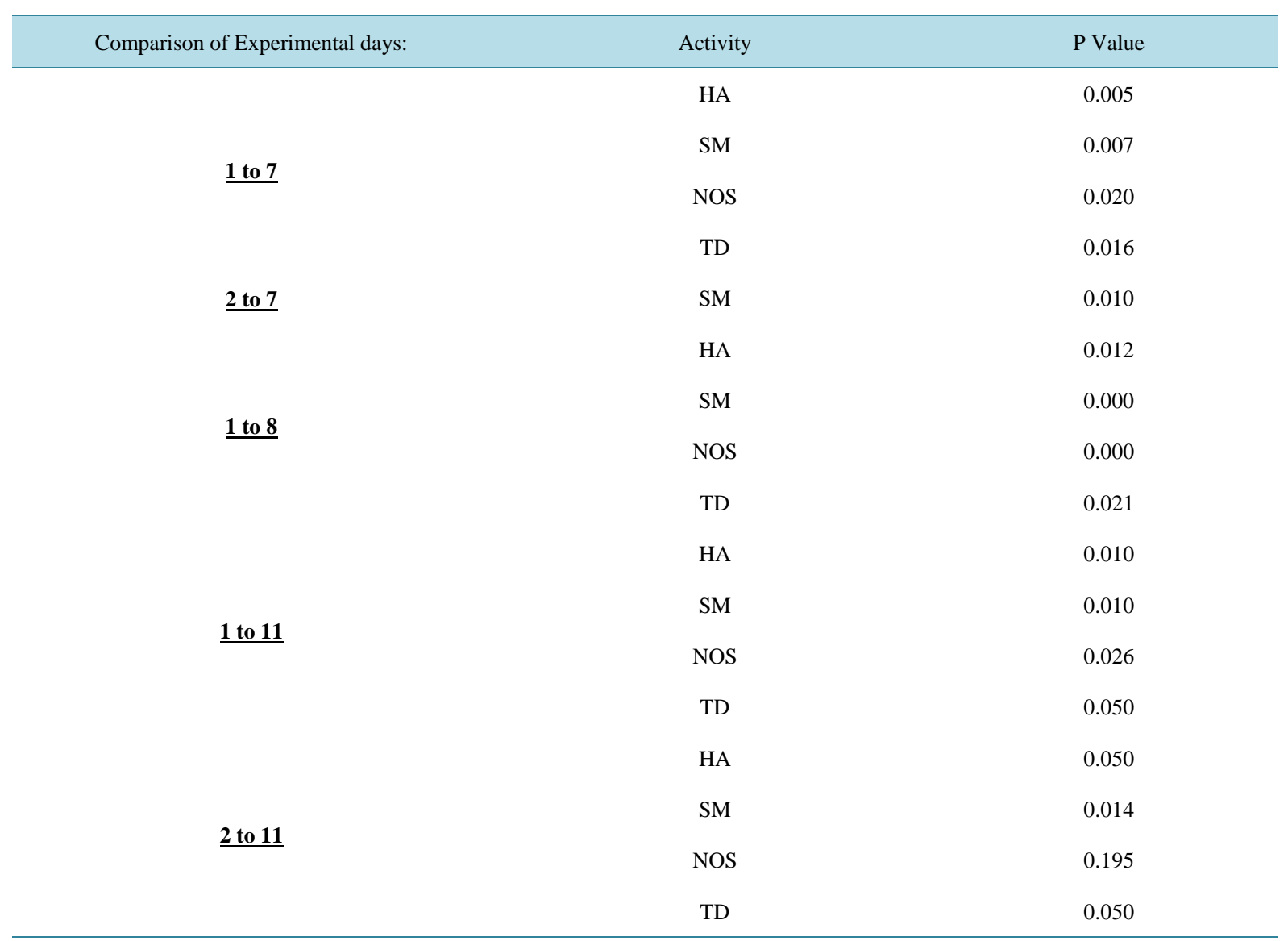

The OASIS program organized these beam interruption recordings into several different locomotor movement indices, such as total distance (TD), which measures the total distance traveled in $\mathrm{cm}$, horizontal activity (HA), which measures the overall locomotor activity in the lowest tier of the field test cage, vertical activity (VA) which measures the specific behavior of rearing and is used to assess the overall amount of locomotor activity, and the number of stereotypic activity (NOS), which counts the number of repetitive movement episodes with at least one second interval before the beginning of another episode of movements [9]-[12] [14] [15] [17] [19]. The HA and the VA were computed as the total numbers of beam interruptions that occurred in the horizontal and vertical plane, respectively. The TD recorded the ambulation in $\mathrm{cm}$, and the NOS were computed as the number of repetitive, purposeless movements. Recording continued non-stop for eleven consecutive days, except for the break window between 06:30 and 07:00 for daily animal handling, such as feeding, weighing, and saline or amphetamine injection. The sound-proof experimental room was locked during recording hours so that the animals were not disturbed.

\subsection{Drug}

Amphetamine sulfate (Sigma) was dissolved in a $0.9 \%$ saline solution to a final concentration of $0.6 \mathrm{mg} / \mathrm{kg}$ amphetamine and dosage was calculated as free base. Previous repetitive amphetamine dose response experiments [9]-[12] [17] showed that this dose $(0.6 \mathrm{mg} / \mathrm{kg}$ i.p.) elicited significant $(\mathrm{p}<0.001)$ behavioral sensitization. Each animal was weighed before injection and all injections were equalized to a volume of $0.8 \mathrm{ml}$. Injections were administered intraperitoneally (i.p.) using a $26 \mathrm{~g}$ needle. Locomotor activity recording was started immediately after injection, one hour into the light phase, until the next morning without interruption for 11 consecutive days except during animal handling between 06:30 and 07:00. All injections were given about 07:00. All efforts were 
made to minimize animal suffering and to reduce the number of animals used for the experiment. The study was conducted according to the declaration of Helsinki and approved by the institutional animal welfare committee. In preliminary experiments, the female rats were kept four per cage from postnatal (P) day 40 to P80 with the intention that all the female rats would cycle on the same day. Vaginal smears [20] were taken daily prior to daily amphetamine injections. It was found that the amphetamine treatment selected in the study did not alter the estrous cycle. In this study, all experiments started in the pre-estrous day and lasted for eleven days i.e., about three estrous cycles.

\subsection{Data Analysis}

All locomotor parameters (indices) were evaluated to test for the drug effects during the $24 \mathrm{~h}$ post-injection period. The locomotor indices recorded included horizontal activity (HA), number of stereotypical movements (NOS), total distance (TD), traveling and stereotypic movement counts (SM). The data comparison was divided into four sections or phases: acute, induction, washout, and expression. The phases were identified as follows: (1) the acute phase compared the data obtained on experimental day 2 (the initial amphetamine injection) with that obtained on experimental day 1 after the saline injection (i.e. control) to observe whether a single acute injection had an effect on locomotor circadian rhythm activity pattern (Table 1); (2) the induction phase compared the activity obtained on experimental day 7 to that observed on experimental day 1 and 2 to determine if 6 days of amphetamine treatment induces an alteration in the circadian rhythm activity pattern; (3) the washout phase consists of the activity level during experimental day 8, 9, and 10 (Table 1) compared to experimental day 1 to observe whether six days of amphetamine treatment changes the baseline circadian rhythm activity pattern when treatment has ceased; and (4) the expression phase utilized the data obtained on experimental day 11 after amphetamine re-challenge (Table 1) compared to the activity pattern on experimental days 1 and 2 to determine whether "chronic" amphetamine treatment effects are expressed after the washout days in order to determine if the amphetamine rechallenge treatment elicited a "long-lasting" effect.

Two calculations were used: (1) 6 of the 10 min consecutive sections (bins) of the locomotor activity indices were summed and averaged to produce hourly histograms with their standard errors. The histograms were used to analyze and compare the locomotor activity visually between the experimental days. (2) The second evaluation used the 10min counts (bins) as points in a time series for statistical analysis using the Cosine Curve Statistical Analysis (CCSA) test [21] to perform statistical comparisons by parameterizing the hourly activity pattern. The CCSA analysis technique group the 24 hour data into three model parameters by estimating the MESOR (Midline estimate of rhythm average activity represented by the curve); Amplitude (distance from the mesor to the highest point-the peak activity), and Acrophase (time at which the maximum amplitude occurs) to model of the cyclic nature of the activity over a 24 hour cycle. The estimates of these three parameters provide the ability to test for statistically significant changes in locomotor activity patterns with respect to time and intensity within the 24 hours of each day. It is therefore possible to determine whether a significant shift in locomotor activity diurnal rhythm pattern occurred in the treatment phases [18] [19] [21]. The CCSA analysis technique was selected because it best evaluated the potential hour to hour magnitude and timing of the 24 hours activity with minimal programmer estimation. All rats in a day-treatment group were graphed as a pair of symbols (i.e. a pair of triangles) for each time intervals which had the same mean and standard deviation as all rats within that day-treatment group interval. In addition, the CCSA tested parameters independently for a null hypothesis of no difference as well as in combinations. The day vs. day contrasts used were selected priorly to enable valid contrast of day pairs for each 24 hour of cyclic data using $3 \mathrm{f}$-tests per day pair. This large number of f-tests was used because several aspects of the long-term behavior were independently evaluated.

\section{Results}

\subsection{Control}

The control (saline) group exhibited the locomotor activity pattern expected for nocturnal animals, with increased activity during the dark phase and decreased activity during the light phase. The circadian rhythm pattern of activity was similar in all experimental days (days 1 to 11) with only minor no significant fluctuations. For example, the experimental day 1 activity pattern following saline injection was similar to the experimental day 5 activity patterns (Figure 1). Thus, any alteration from the activity pattern observed on experimental day one can and was attributed to the effects of amphetamine. 
HA

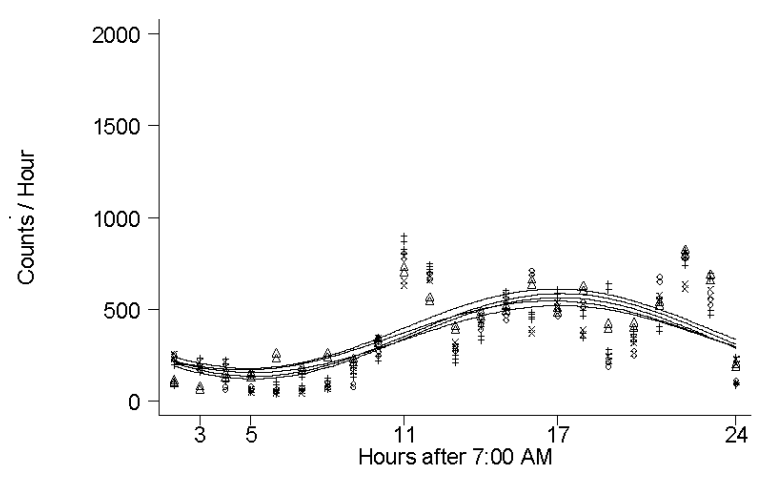

(a)

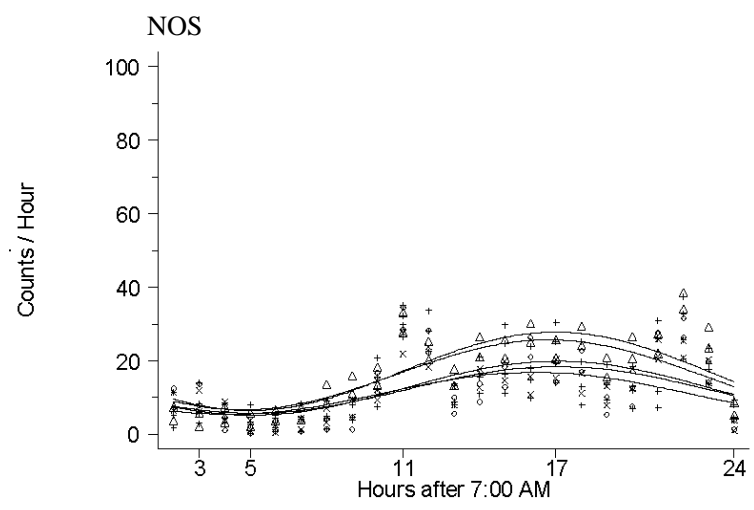

(c)

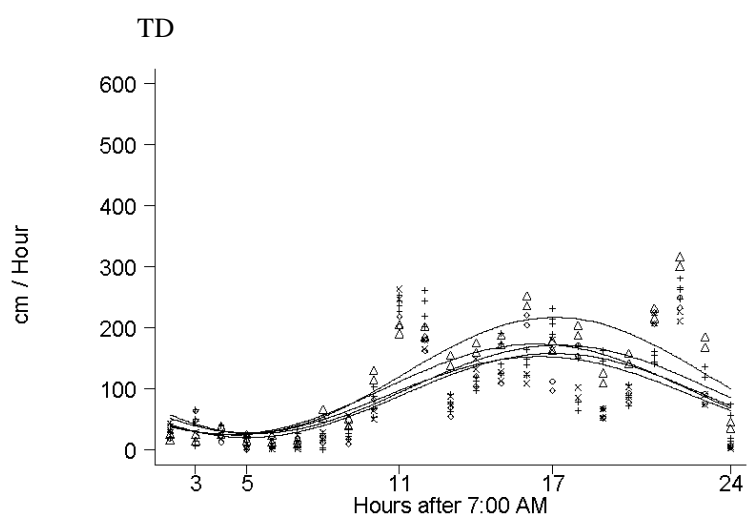

(b)

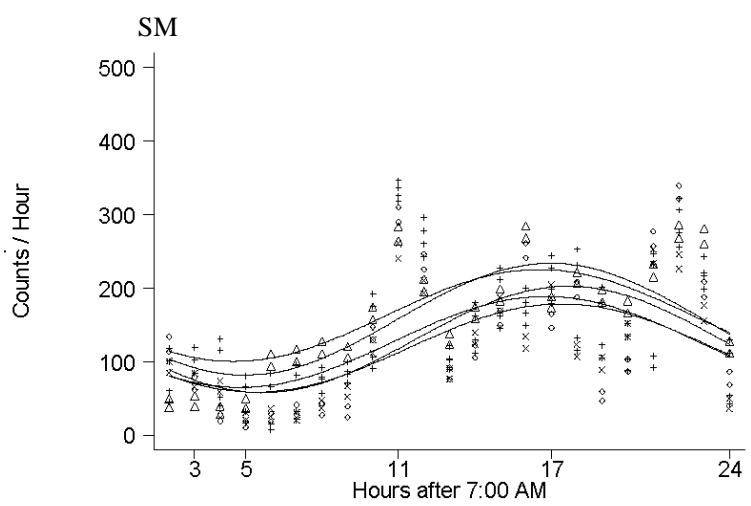

(d)

Figure 1. Cosine super impose plots comparing the activity indices: (a) horizontal activity (HA), (b) total distance (TD), (c) number of stereotypic movements (NOS), and (d) stereotypic counts (SM) for the controls on experimental day 1, 2, 7, 8, and 11 . The symbol pairs: $+; \Delta ; \mathrm{x} ; 0 ; 23$ and, $\cdot$ indicate the mean and the standard error (SE) of each of the data point of the experimental day $1,2,7,8$, and 11 respectively showing that the activity exhibits the same pattern in all experimental days.

\subsection{Acute Effect of Amphetamine: Experimental Day 2 vs. Experimental Day 1}

Amphetamine ( $0.6 \mathrm{mg} / \mathrm{kg}$ i.p.) treatment elicited increases in all of the locomotor indices (HA, TD, NOS, SM) for the first two hours after the injection (Figure 2) followed by a slight decrease in activity during the light phase of experimental day 2 compared to experimental day 1 as indicated by the hourly histogram. In each experimental day at least two increases of activity occurs; one immediately post injection and the second when the illumination of the room changes from light to dark. However, when this observation was analyzed by the CCSA test the above changes were not statistically significant. The activity level of all four of the locomotor indices showed a similar activity pattern during the dark phase of experimental day 2 compared to experimental day 1 (Figure 2).

\subsection{Repetitive Effect of Amphetamine-Induction Phase}

(a). Comparing Experimental day 7 vs. experimental day 1

By experimental day 7, the animals have received 6 consecutive daily amphetamine treatments (i.e. experimental day 2 to 7). The hourly histograms of experimental day 7 showed an increase in activity in all of the locomotor indices (HA, 10TD, NOS, and SM) during the first three hours post-injection compared to experimental day 1 . The activity returned to baseline five to six hours after the injection followed by an increase in activity during the dark phase on day experimental 7 compared to experimental day 1 . The CCSA test indicated that the mesor parameters of HA, TD, NOS, and SM changed significantly $(\mathrm{p}=0.005,0.016,0.02$, and 0.007 , respectively) (Table 2) on experimental day 7 compared to experimental day 1. 


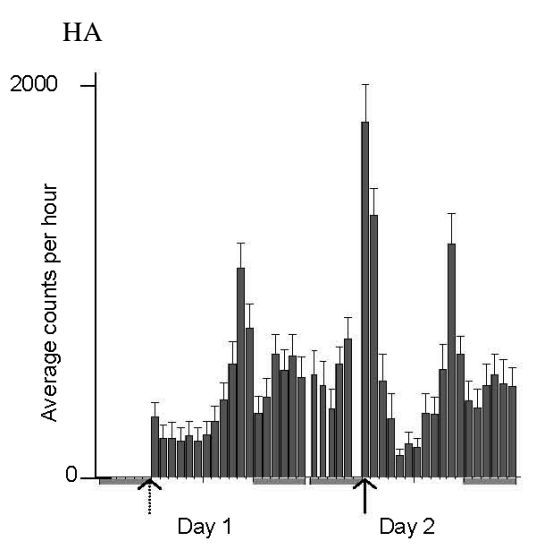

(a)

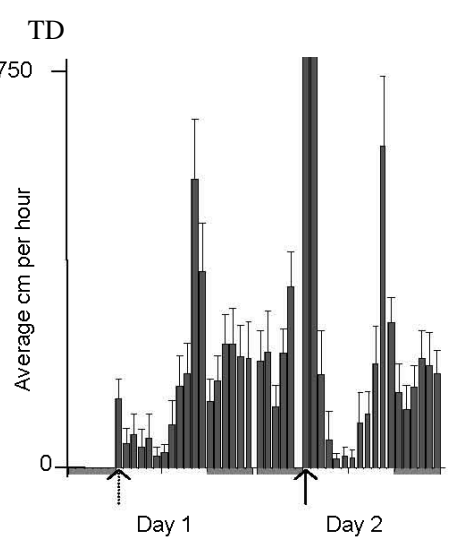

(b)

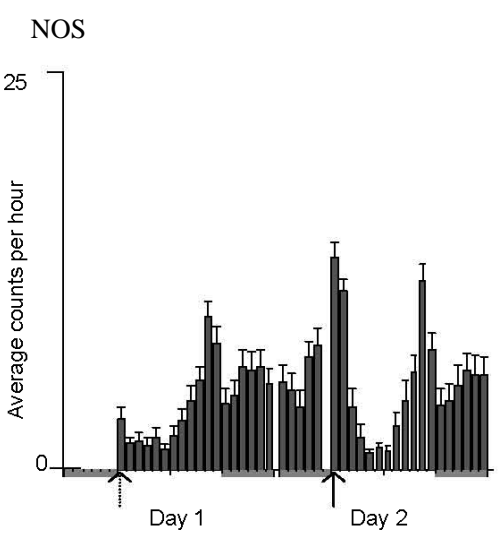

(c)

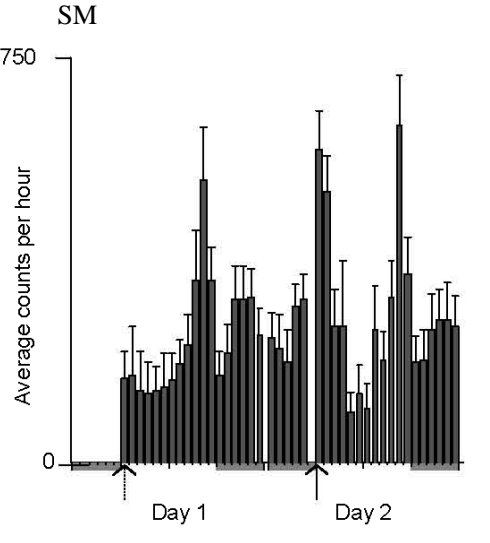

(d)

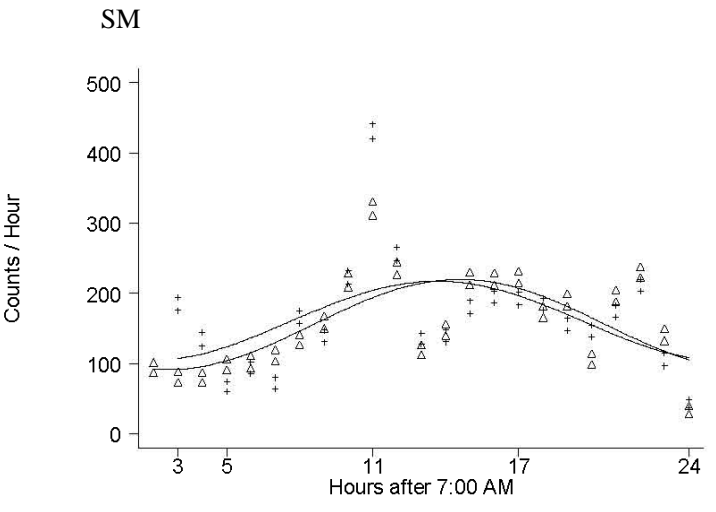

(e)

Figure 2. Hourly histograms of the activity indices a) HA, b) TD, c) NOS, d) SM from experimental day 1 and experimental day 2 (acute effect), and e) SM activity plot indicating no significant difference in the mesor parameter. Recording began at 7:00 AM on day 1 . The dashed and solid arrows represent the time of saline and amphetamine injection (7:00 AM), respectively. The symbols + and $\Delta$ indicate the mean and the SE of each of the data point of experimental day 1 and 2 respectively. The shaded area under the histograms indicate the dark (night) period; the arrow indicates the time of injection.

Table 2. P values for the mesor parameter of circadian rhythm activity indices found significant using CCSA statistical analysis. Days 2 to 7: shows the comparison between the first amphetamine administration (experimental day 2) with the amphetamine given at experimental day 7. Day 1 to 8: shows the comparison between the control recording at day 1 to the first washout day; days 2 to 11 and 1 to 11: Compares the observation of experimental day 1 and 2 with the observation obtained after the last amphetamine exposure (see Table 1).

\begin{tabular}{cccccc}
\hline & N & \multicolumn{2}{c}{ Experimental Day } \\
\hline & & day 1 & days 2-7 & days 8-10 & day 11 \\
I & 12 & Saline & Saline & Washout & Amphetamine \\
II & 12 & Saline & Amphetamine & Washout & Ame \\
\hline
\end{tabular}

(b). Comparing Experimental day 7 vs. experimental day 2

The hourly histograms showed increased TD, HA, SM, and NOS activities during the first hour after amphetamine administration on experimental day 7 compared to experimental day 2 as well as an increase in their activities during the dark phase (Figure 3). Comparison of the activity patterns by the CCSA test showed that only the SM activity exhibited a statistically significant change in the mesor parameter $(p=0.01)$ exhibited increases in the activity and the peak amplitude shift to the right of the circadian rhythm pattern on experimental day 7 compared to experimental day 2 (Table 2 and Figure 3). 


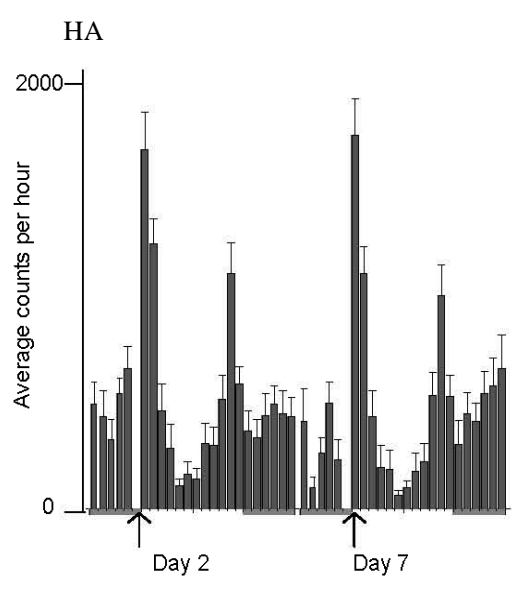

(a)
TD

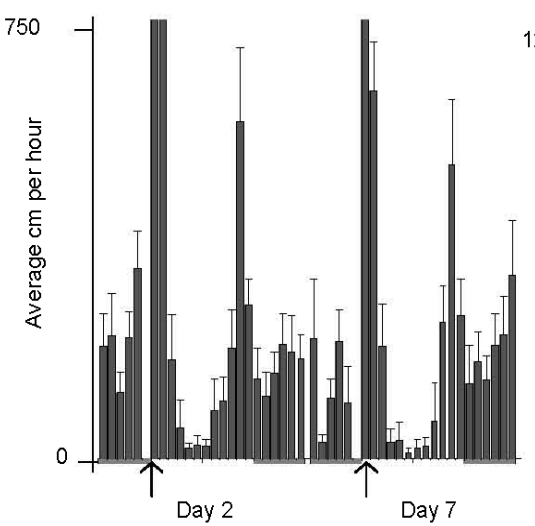

(b)
NOS

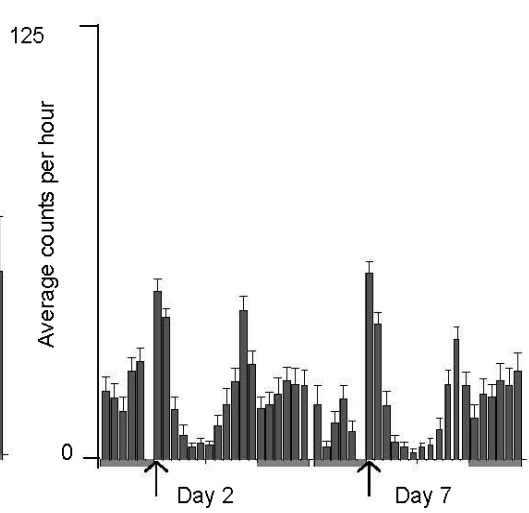

(c)

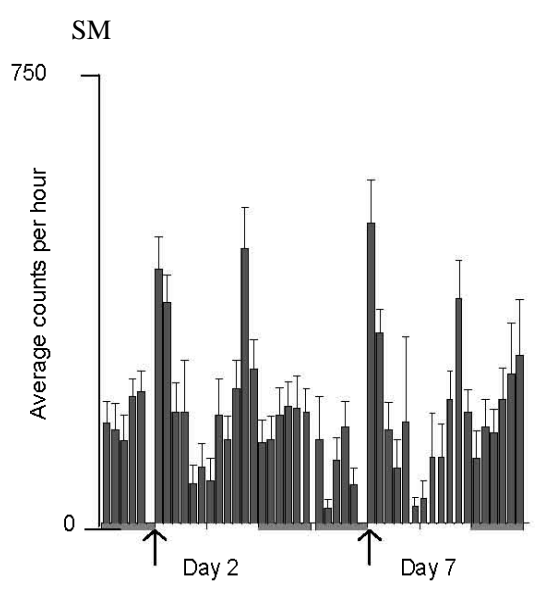

(d)

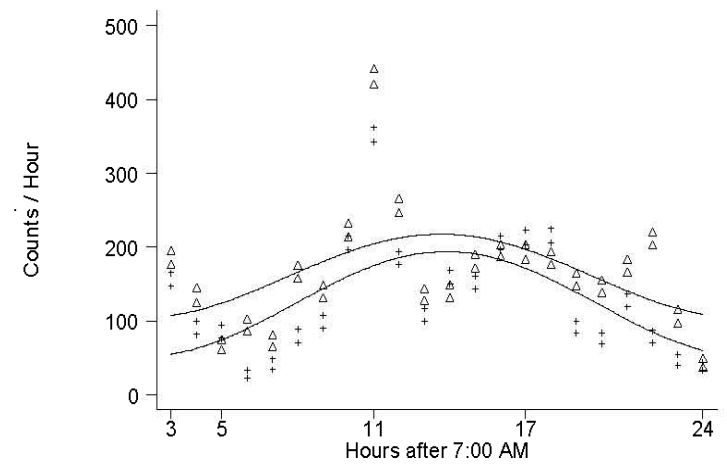

(e)

Figure 3. Hourly histogram comparing the activity indices a) HA, b) TD, c) NOS, d) SM from experimental day 2 and experimental day 7, and e) SM activity plot indicating significance in the mesor parameter. The arrows indicate the time of injection (7:00AM). The symbols + and $\Delta$ indicate the mean and the SE of each of the hourly data points of experimental day 2 and 7 respectively. The shaded area under the histograms indicate the dark (night) period; the arrow indicates the time of injection.

\subsection{Amphetamine Re-Challenge Expression Phase}

1) Comparing Experimental day 1 vs. experimental day 11

Comparing the locomotor activity of experimental day 1 to that obtained at experimental day 11 was used to determine whether any alteration in the circadian activity occurred after six daily amphetamine exposure and three washout days. All four indices (HA, TD, NOS, and SM) exhibited more activity during the first two hours post injection after amphetamine re-challenge as well as during the dark phase on experimental day 11 compared to experimental day 1 . There were also11 a return to baseline activity during the light phase on experimental day 11 compared to experimental day 1 . When these activity patterns were analyzed using the CCSA test, the mesor parameter of HA, TD, NOS, and SM exhibited significant changes on experimental day 11 compared to experimental day $1(\mathrm{p}=0.01,0.01,0.026$ and 0.05 , respectively) (Table 2).

2) Comparing Experimental day 11 vs. experimental day 2

Comparing experimental day 11 to experimental day 2 was used to assess whether any alteration in the circadian locomotor activity pattern after chronic amphetamine administration at experimental day 11 occurs compared to the initial amphetamine effect on experimental day 2. The locomotor activities of experimental day 11 compared to experimental day 2 showed more activity during the first two hours post injection in all four locomotor indices (HA, NOS, SM, TD) (Figure 4). There was a decrease during the light phase in the locomotor 


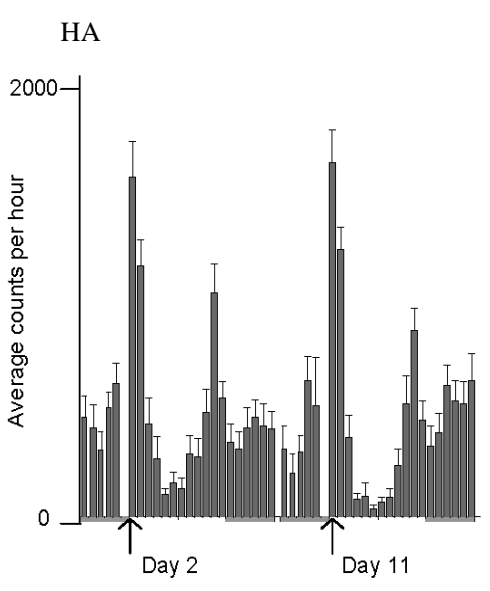

(a)

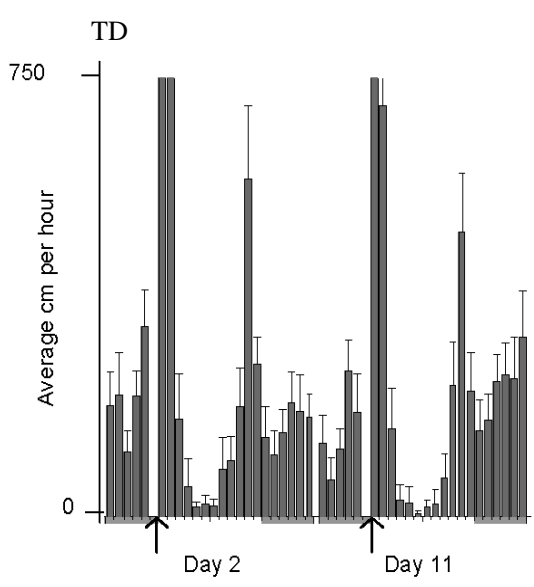

(b)

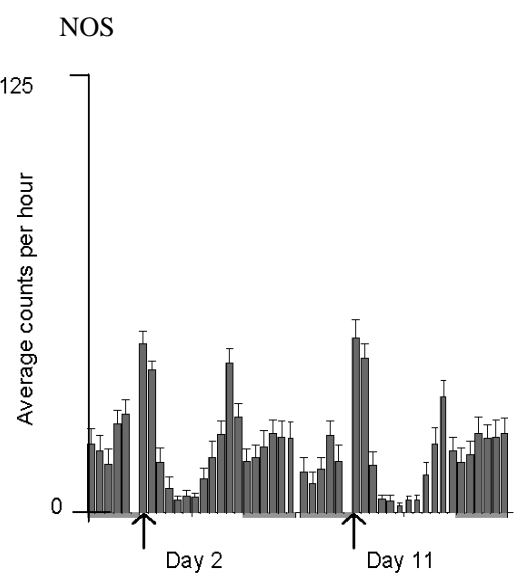

(c)

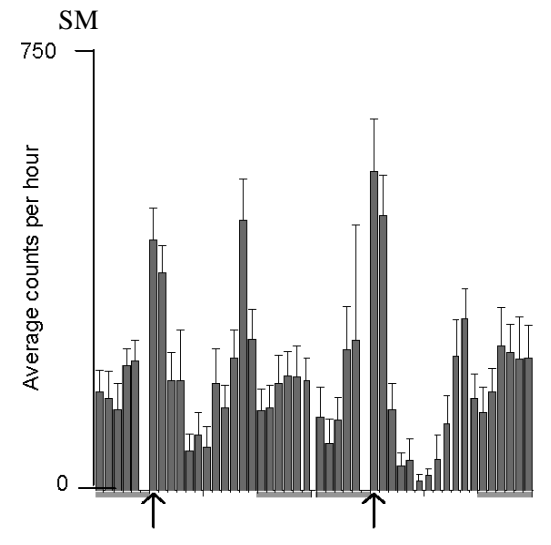

(d)

$$
\mathrm{SM}
$$

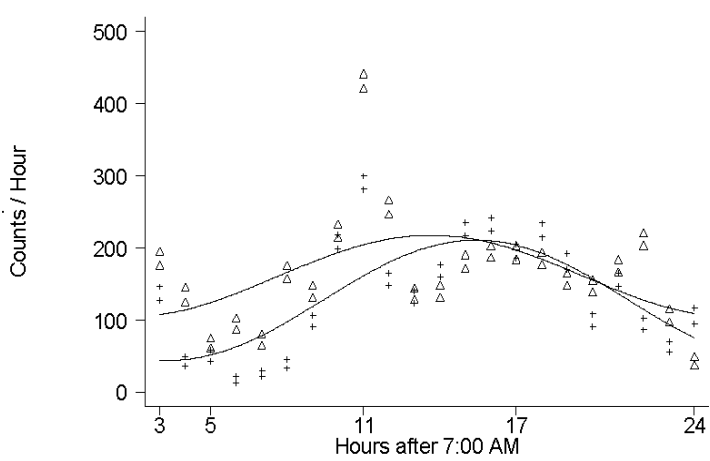

(e)

Figure 4. Hourly histogram comparing the activity indices a) HA, b) TD, c) NOS, d) SM from experimental day 2 and experimental day 11, e) is the CSSA data of SM activity, the plot indicates significance in the mesor parameter. The arrows indicate the time of injection (7:00AM). The symbols + and $\Delta$ indicate the mean and the SE of each of the hourly data points of experimental day 2 and 11 respectively. The shaded area under the histograms indicate the dark (night) period; the arrow indicates the time of injection.

circadian activity rhythm patterns of HA and SM in experimental day11 compared to experimental day 2 while the NOS and TD activities remained similar on experimental day 11 and 2. During the dark phase, the HA, TD, and SM activities increased on experimental day 11compared to experimental day 2 while NOS activity remained similar to that on experimental day 2 (Figure 4). The statistical analysis of these observations using the CCSA test revealed significant changes in locomotor circadian activity rhythm patterns of SM in the mesor parameter ( $\mathrm{p}=0.014$; Table 2$)$ and the acrophase shifted significantly to the right.

\subsection{Washout Phase: Comparing Experimental Days 8, 9, and 10 vs. Experimental Day 1}

All four locomotor indices (HA, SM, NOS, and TD) exhibited more locomotor activity during the first two morning light phase hours followed by a three to four hour decrease in activity on experimental days 8 , 9, and 10 compared to experimental day 1 . All four indices also showed a decrease in activity during the dark phase on experimental day 8compared to experimental day 1 (Figure 5). These changes in the locomotor indices persisted from experimental day 8 to experimental day 10 (Figure 6).

Statistical analysis of experimental day 8 to experimental day 1 using CCSA testing showed that the HA, SM, NOS, and TD activity exhibited significant changes in circadian locomotor rhythm pattern $(p=0.012,0.000$, 0.000 , and 0.021 , respectively), the mesor amplitude was attenuated and the acrophase shift to the right (Figure 5 and Figure 6). 
HA

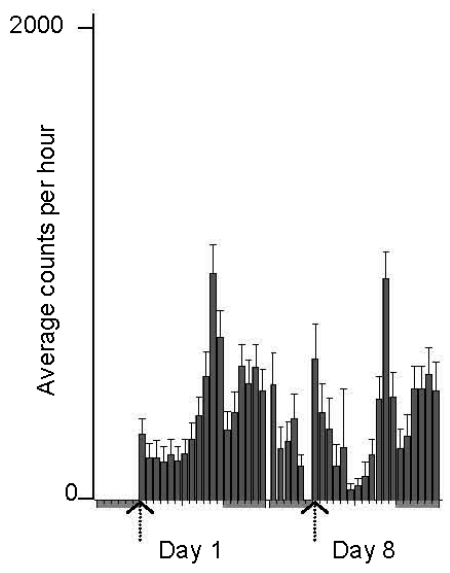

(a)
TD

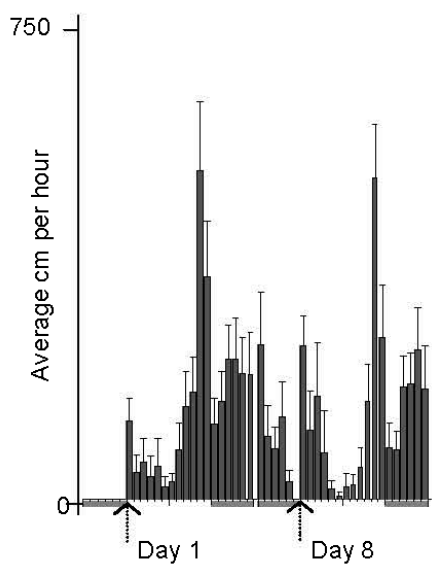

(b)
NOS

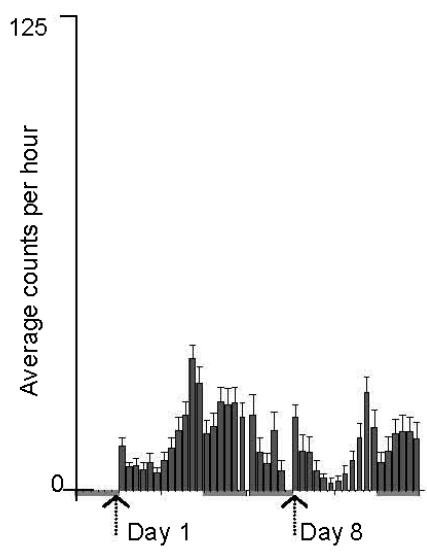

(c)

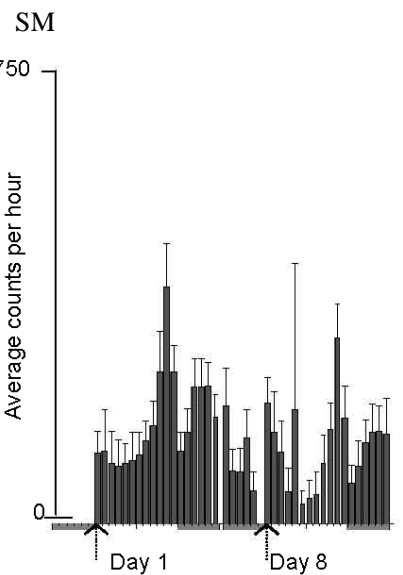

(d)

SM

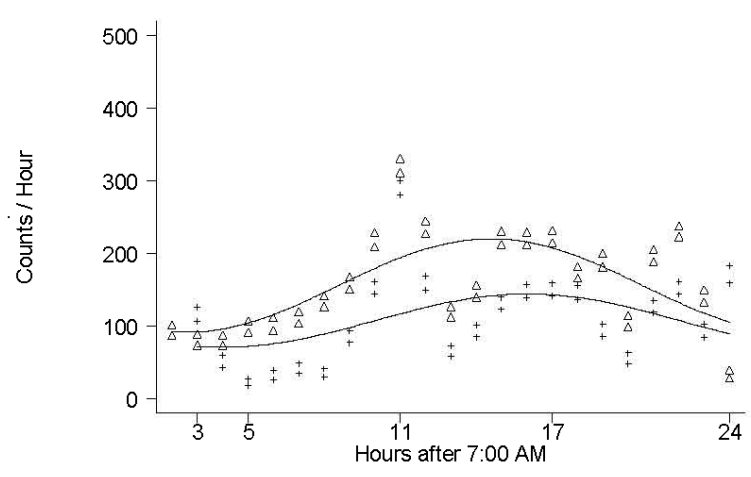

(e)

Figure 5. Hourly histogram comparing the activity indices a) HA, b) TD, c) NOS, d) SM from experimental day 1 and experimental day 8, and e) SM activity plot indicating significance in the mesor parameter. Dashed arrows represent the time of saline injection. The symbols + and $\Delta$ indicate the mean and the SE of each of the hourly data points of experimental day 1 and 8 respectively. The shaded area under the histograms indicate the dark (night) period; the arrow indicates the time of injection.

\section{Discussion}

Circadian rhythms are internal biological rhythms typically occurring over a 24-hour period. These rhythms govern the sleep cycle as well as other vital functions such as heart rate, blood pressure, body temperature, and hormone production. These internal factors along with light exposure and other external environmental cues allow humans (as well as many other organisms) to anticipate regular changes in the environment. The 24-hour cycle which coincides with the rotation of the earth is governed by specific neural pathways and a set of neural structures. Several components play a key role in the regulation of these structures and pathways. The master pacemaker or "clock" of the human body lies in the suprachiasmatic nucleus (SCN), located in the anterior portion of the hypothalamus [7] [22]-[26]. Any disturbance in the internal circadian rhythm pattern can have extensive effects on many physiological functions such as sleep, temperature regulation, and hormone production to name a few. Many drugs and medications including psychostimulants show varying pharmacokinetics over the course of a 24 hour day [9]-[11] [14]-[17]. Prolonged drug use can produce tolerance resulting in the requirement for an increased amount of the drug to elicit the same response or sensitization (i.e. reverse tolerancesensitization is an enhanced behavioral response as a result of repeated drug exposure), a phenomenon that can lead to drug dependence 


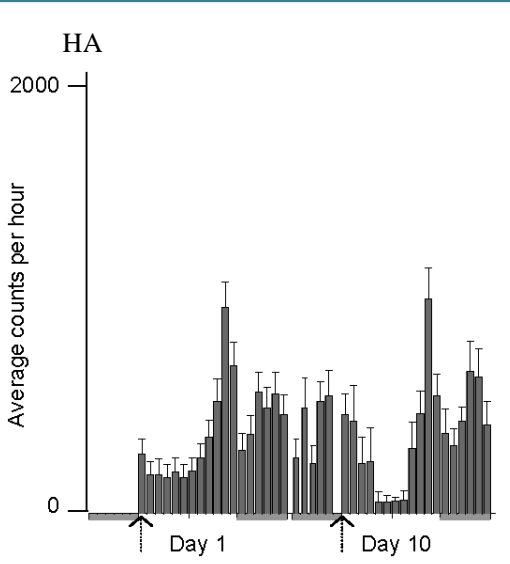

(a)

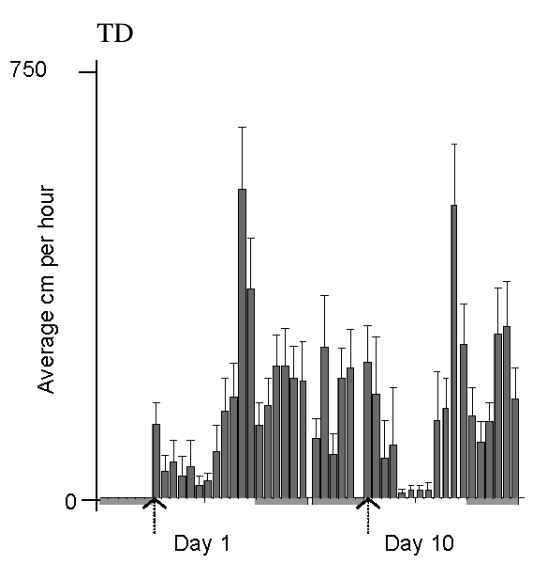

(b)

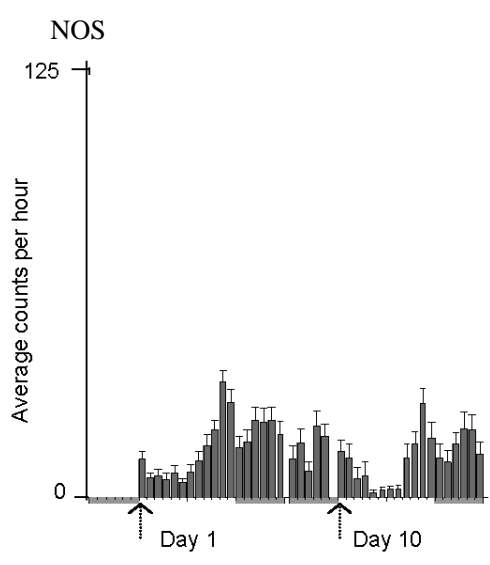

(c)

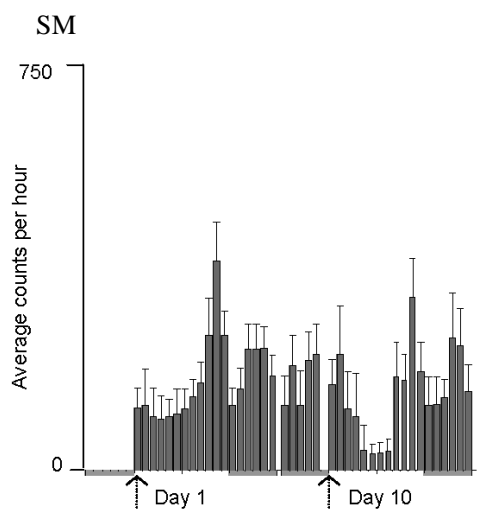

(d)

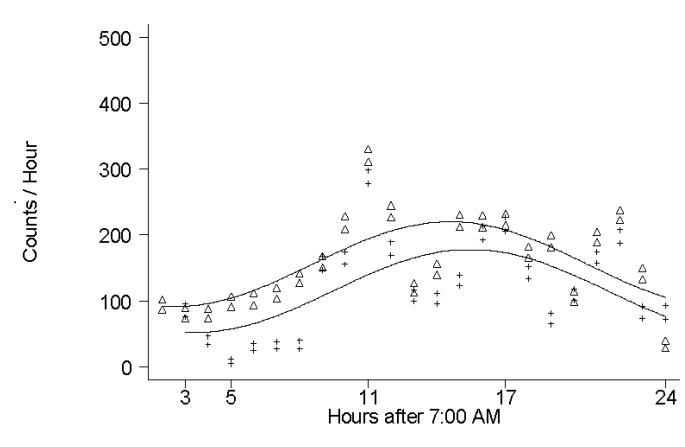

(e)

Figure 6. Hourly histogram comparing the activity indices a) HA, b) TD, c) NOS, d) SM from experimental day 1 and experimental day 10, and e) SM activity plot indicating significance in the mesor parameter. Dashed arrows represent the time of saline injection. The symbols + and $\Delta$ indicate the mean and the SE of each of the hourly data points of experimental day 1 and 10 respectively. The shaded area under the histograms indicate the dark (night) period; the arrow indicates the time of injection.

[7] [27]-[29]. Amphetamine treatment at 07:00, 13:00, 19:00 and 01:00 elicits behavioral sensitization at all four times of injection [9]-[11] [17] while methylphenidate given at the same four injection times elicits behavior sensitization only when given during the light (07:00 and 13:00) period [12].

It has been shown that amphetamine induces an increase in DA secretion from both vesicular stores and from nerve terminals [30]. Amphetamine also binds to DA transporters preventing DA reuptake and thus, increases extracellular DA in the synaptic cleft [31]. It was reported that drugs that can modulate the central nervous system are able to alter gene expression and modify the expression of clock genes, resulting in an alteration in the circadian rhythm pattern [8]. The relationship between amphetamine and alteration of striatal gene expression is thought to elicit long-term changes in behavior including sensitization, tolerance, and/or dependence produced by repetitive amphetamine use [32]. Studies suggest that psychostimulant treatment may alter the sensitivity of presynaptic DA receptors, and this increase in DA sensitivity results in sensitization as well as cross sensitization with other psychostimulants. This cross sensitization between psychostimulants and other medications increases the potential for drug dependence later in life [33].

Since the final path of circadian activity is regulated by the clock genes, changes in clock gene expression are a result of long-term effects on clock machinery. This leads us to hypothesize that drug induced changes in the circadian rhythm activity pattern indicate long term effects of the drug. In the present study, 11 consecutive days of locomotor activity with and without amphetamine treatment were recorded and the results were statistically analyzed using the cosine curve statistical analysis (CCSA) test to determine whether amphetamine treatment elicited significant changes in circadian rhythm activity pattern. Changes in circadian rhythm activity pattern 
following pharmacotherapy treatment would indicate that the treatment elicited a long term effect. Multiple activity indices were utilized in order to study any aspect change in the locomotor circadian rhythm activity pattern. While CCSA analysis indicated that the locomotor circadian rhythm patterns of HA, TD, SM, and NOS were not changed after a single injection of $0.6 \mathrm{mg} / \mathrm{kg}$, while after six days of amphetamine administration (i.e. experimental day 2 to 7), 3 washout days, and the amphetamine re-challenge at (i.e. experimental day 11), statistically significant changes in activity patterns were observed.

Changes in circadian rhythm on experimental day 7 and experimental day 11compared to experimental day 2 exhibited two phenomena. An increase in activity on experimental day 7 and experimental day 11 compared to experimental day 2 were observed, this increase in activity suggests: 1) that sensitization was established and 2) a significant change in circadian rhythm activity pattern took place. One possible explanation is that the development of sensitization resulted in an alteration in the circadian rhythm activity pattern or the reversed, change in the circadian activity pattern as a result of modulation of cellular, molecular transcription factors and clock genes that result in sensitization [34] [35]. However, whether sensitization results in an alteration in circadian rhythm or circadian rhythm alteration leads to sensitization requires further consideration.

An additional point of interest is the significant change in locomotor circadian rhythm activity pattern during the washout days. The animals showed an increase inactivity at the time of injection on the previous days followed by a decrease in daytime activity and a decrease in nighttime activity compared to pre-amphetamine treatment. This increase in activity can be explained due to anticipatory behavior which persists for several days after repetitive injections of the drug [36] or as withdrawal behavior due to previous 6 days of amphetamine treatment followed by an abrupt treatment [18] [19] [37]. The variation in night time circadian rhythm activity pattern correlates with reports of insomnia linked to psychostimulant treatment with ADHD and other drugs of abuse [38] [39]. These changes indicate that chronic amphetamine administration leads to a long-term alteration in behavior and circadian rhythm with a further increase in the behavioral effect elicited by the same dosage of amphetamine administered due to, sensitization, compared to the response produced by acute amphetamine treatment. The disruption in the circadian rhythm activity patterns may indicate molecular changes due to chronic psychostimulant administration on circadian rhythm gene transcription. This may have implications for other circadian rhythm regulated processes [40]-[42]. Indeed molecular studies reported that repetitive exposure to psychostimulant upregulates the cAMP second messenger pathway. This regulation results in activation of transcription factor cAMP response element binding protein (CREB) as well as the transcription factor $\Delta$ Fos $B$ [34] [35]. These studies indicate that repeated exposure to psychostimulants alter the amount of gene expression in the brain. In addition, it was reported that psychostimulant exposure induces structural plasticity that is responsible for long-term behavioral plasticity [43]. All the above molecular and structural plasticity suggest that the repetitive exposure of psychostimulant elicits long term effects.

\section{Conclusion}

In conclusion, these data suggest that "chronic" amphetamine administration in female WKY rats leads to longterm effects presumably due to molecular effects on the circadian clock genes as demonstrated by the significant alteration in circadian rhythm activity pattern.

\section{Acknowledgements}

The authors wish to thank Catherine M. Claussen, D. Wood and Drs. Julia Lever and Alan Levin for helping with the manuscript preparation. This research was supported in part by NIH DA 14441 and R01-DA027222 grants.

\section{References}

[1] Hechtman, L. (2000) Assessment and Diagnosis of Attention-Deficit/Hyperactivity Disorder. Child \& Adolescent Psychiatric Clinics of North America, 9, 481-498.

[2] Mackworth, J.F. (1965) The Effect of Amphetamine on the Detectability of Signals in a Vigilance Task. Canadian Journal of Psychology, 19, 104-110. http://dx.doi.org/10.1037/h0082897

[3] Evenden, J.L. and Robbins, T.W. (1985) The Effects of $d$-Amphetamine, Chlordiazepoxide and Alpha-Flupenthixol on Food-Reinforced Tracking of a Visual Stimulus by Rats. Psychopharmacology, 85, 360-366.

http://dx.doi.org/10.1007/BF00428202 
[4] Toon, S., Holt, B.L., Langley, S.J., Mullins, F.G.P., Rowland, M. and Halliday, M.S. (1990) Pharmacokinetic and Pharmacodynamic Interaction between the Antidepressant Tianeptine and Oxazepam at Steady-State. Psychopharmacology, 101, 226-232. http://dx.doi.org/10.1007/BF02244131

[5] Gray, J.D., Punsoni, M., Tabori, N.E., Melton, J.T., Fanslow, V., Ward, M.J., Zupan, B., Menzer, D., Rice, J., Drake, C.T., Romeo, R.D., Brake, W.G., Toress-Reveron, A. and Milner, T.A. (2007) Methylphenidate Administration to Juvenile Rats Alters Brain Areas Involved in Cognition, Motivated Behaviors, Appetite, and Stress. Journal of Neuroscience, 27, 7196-207. http://dx.doi.org/10.1523/JNEUROSCI.0109-07.2007

[6] Scheving, L.E., Vedral, D.F. and Pauly, J.E (1968) Daily Circadian Rhythm in Rats to Damphetamine Sulphate: Effect of Binding and Continuous Illumination on the Rhythm. Nature, 219, 621-622. http://dx.doi.org/10.1038/219621a0

[7] Uz, T., Akhisaroglu, M., Ahmed, R. and Manev, H. (2003) The Pineal Gland Is Critical for Circadian Period1 Expression in the Striatum and for Circadian Cocaine Sensitization in Mice. Neuropsychopharmacology, 28, 2117-23.

[8] Uz, T., Ahmed, R., Akhisaroglu, M., Kurtuncu, M., Imbesi, M., DirimArslan, A. and Manev, H. (2005) Effect of Fluoxetine and Cocaine on the Expression of Clock Genes in the Mouse Hippocampus and Striatum. Neuroscience, 134, 1309-1316. http://dx.doi.org/10.1016/j.neuroscience.2005.05.003

[9] Gaytan, O., Ghelani, D., Martin, S., Swann, A. and Dafny, N. (1996) Dose-Response Characteristics of Methypehedate on Different Indices of Rats Locomotor Activity at the Beginning of the Dark Cycle. Brain Research, 727, 13-21. http://dx.doi.org/10.1016/0006-8993(96)00296-X

[10] Gaytan, O., Swann, A.C. and Dafny, N. (1996) Effects of Amphetamine at the Beginning of the Light Cycle on Multiple Indices of Motor Activity in the Rat. European Journal of Pharmacology, 300, 1-8.

[11] Gaytan, O., Swann, A. and Dafny, N. (1998) Diurnal Differences in Rat's Motor Response to Amphetamine. European Journal of Pharmacology, 345, 119-128. http://dx.doi.org/10.1016/S0014-2999(97)01558-6

[12] Gaytan, O., Yanga, P., Swann, A. and Dafny, N. (2000) Diurnal Differences in Sensitization to Methylphenidate. Brain Research, 864, 24-39. http://dx.doi.org/10.1016/S0006-8993(00)02117-X

[13] Melnick, S.M. and Dow-Edwards, D.L. (2001) Differential Behavioural Responses to Chronic Amphetamine in Adult Male and Female Rats Exposed to Postnatal Cocaine Treatment. Pharmacology, Biochemistry, and Behavior, 69, 219224. http://dx.doi.org/10.1016/S0091-3057(01)00545-7

[14] Yang, P.B., Swann, A.C. and Dafny, N. (2003) Chronic Pretreatment with Methylphenidate Induces Cross-Sensitization with Amphetamine. Life Sciences, 73, 2899-2911. http://dx.doi.org/10.1016/S0024-3205(03)00673-8

[15] Yang, P.B., Swann, A.C. and Dafny, N. (2006) Acute and Chronic Methylphenidate Doseresponse Assessment on Three Adolescent Male Rat Strains. Brain Research, 71, 301-310.

[16] Dafny, N. and Yang, P.B. (2006) The Role of Age, Genotype, Sex, and Route of Acute and Chronic Administration of Methylphenidate: A Review of Its Locomotor Effects. Brain Research Bulletin, 68, 393-405. http://dx.doi.org/10.1016/j.brainresbull.2005.10.005

[17] Gaytan, O., Lewis, C., Swann, A. and Dafny, N. (1999) Diurnal Differences in Amphetamine Sensitization. European Journal of Pharmacology, 374, 1-9. http://dx.doi.org/10.1016/S0014-2999(99)00243-5

[18] Algahim, M.F., Yang, P.B., Wilcox, V.T., Burau, K.D., Swann, A.C. and Dafny, N. (2009) Prolonged Methylphenidate Treatment Alters the Behavioral Diurnal Activity Pattern of Adult Male Sprague-Dawley Rats. European Journal of Pharmacology, 92, 93-99. http://dx.doi.org/10.1016/j.pbb.2008.10.021

[19] Lee, M.J., Yang, P.B., Wilcox, V.T., Burau, K.D., Swann, A.C. and Dafny, N. (2009) Does Repetitive Ritalin Injection Produce Long-Term Effects on SD Female Adolescent Rats? Neuropharmacology, 57, 201-207. http://dx.doi.org/10.1016/j.neuropharm.2009.06.008

[20] Dafny, N. and Terkel, J. (1990) Hypothalamic Neuronal Activity Associated with Onset of Pseudopregnancy in the Rat. Neuroendocrinology, 51, 459-467. http://dx.doi.org/10.1159/000125375

[21] Bingham, C., Arbogast, B., Guillaume, G.C., Lee, J.K. and Halberg F. (1982) Inferential Statistical Methods for Estimating and Comparing Cosinor Parameters. Chronobiologia, 9, 397-439.

[22] Kurtuncu, M., Arslan, A., Akhisaroglu, M., Manev, H. and Uz, T. (2004) Involvement of the Pineal Gland in Diurnal Cocaine Reward in Mice. European Journal of Pharmacology, 489, 203-205. http://dx.doi.org/10.1016/j.ejphar.2004.03.010

[23] Moore, R.Y. (1983) Organization and Function of a Central Nervous System Circadian Oscillator: The Suprachiasmatic Hypothalamic Nucleus. Federation Proceedings, 42, 2783-2789.

[24] Minors, D.S. and Waterhouse, J.M. (1986) Circadian Rhythms and Their Mechanisms. Experientia, 42, 1-13.

[25] Reppert, S.M. and Weaver, D.R. (2002) Coordination of Circadian Timing in Mammals. Nature, 418, 935-941. http://dx.doi.org/10.1038/nature00965

[26] Gachon, F., Nagoshi, E., Brown, S.A., Ripperger, J. and Schibler, U. (2004) The Mammalian Circadian Timing System: 
From Gene Expression to Physiology. Chromosoma, 113, 103-112. http://dx.doi.org/10.1007/s00412-004-0296-2

[27] Brandon, C.L., Marinelli, M., Baker, L.K. and White, F.J. (2001) Enhanced Reactivity and Vulnerability to Cocaine Following Methylphenidate Treatment in Adolescent Rats. Neuropsychopharmacology, 25, 651-661. http://dx.doi.org/10.1016/S0893-133X(01)00281-0

[28] Segal, D.S. and Kuczenski, R. (2006) Human Methamphetamine Pharmacokinetics Simulated in the Rat: Single Daily Intravenous Administration Reveals Elements of Sensitization and Tolerance. Neuropsychopharmacology, 31, 941-955. http://dx.doi.org/10.1016/S0893-133X(01)00281-0

[29] Barrett, R.J., Caul, W.F. and Smith, R. (2005) Withdrawal, Tolerance, and Sensitization to Dopamine Mediated Interoceptive Cues in Rats Trained on a Three-Lever Drug-Discrimination Task. Pharmacology Biochemistry and Behavior, 81, 1-8. http://dx.doi.org/10.1016/j.pbb.2004.12.012

[30] Fleckenstein, A.E. and Hanson, G.R. (2003) Impact of Psychostimulants on Vesicular Monoamine Transporter Function. European Journal of Pharmacology, 479, 283-289. http://dx.doi.org/10.1016/j.ejphar.2003.08.077

[31] Pontieri, F.E., Tanda, G. and Chiara, G.D. (1995) Intravenous Cocaine, Morphine, and Amphetamine Preferentially Increase Extracellular Dopamine in the "Shell” as Compared with the "Core" of the Rat Nucleus Accumbens. Proceedings of the National Academy of Sciences of the United States of America, 92, 12304-12308. http://dx.doi.org/10.1073/pnas.92.26.12304

[32] Paulson, P.E. and Robinson, T.E. (1996) Regional Differences in the Effects of Amphetamine Withdrawal on Dopamine Dynamics in the Striatum. Analysis of 21 Circadian Patterns Using Automated Online Microdialysis. Neuropsychopharmacology, 14, 325-337. http://dx.doi.org/10.1016/0893-133X(95)00141-Y

[33] Shuster, L., Hudson, J., Anton, M. and Righi, D. (1982) Sensitization of Mice to Methylphenidate. Psychopharmacologia, 77, 31-36. http://dx.doi.org/10.1007/BF00436096

[34] Chao, J. and Nestler, E.J. (2004) Molecular Neurobiology of Drug Addiction. Annual Review of Medicine, 55, 113132. http://dx.doi.org/10.1146/annurev.med.55.091902.103730

[35] Nestler, E.J. (2004) Molecular Mechanisms of Drug Addiction. Neuropharmacology, 47, 24-32. http://dx.doi.org/10.1016/j.neuropharm.2004.06.031

[36] Kosobud, A., Gillman, A., Leffel, J., Pecoraro, N., Rebec, G. and Timberlake, W. (2007) Drugs of Abuse Can Entrain Circadian Rhythms. The Scientific World Journal, 7, 203-212. http://dx.doi.org/10.1100/tsw.2007.234

[37] White, W. and White, I.M. (2006) An Activity Indicator of Acute Withdrawal Depends on Amphetamine Dose in Rats. Physiology \& Behavior, 87, 368-376. http://dx.doi.org/10.1016/j.physbeh.2005.10.009

[38] Gordon, H. (2007) Sleep, Circadian Rhythm, and Drug Abuse. The Scientific World Journal, 7, 191-193. http://dx.doi.org/10.1100/tsw.2007.227

[39] McClung, C. (2007) Circadian Rhythms, the Mesolimbic Dopaminergic Circuit, and Drug Addiction. The Scientific World Journal, 7, 194-202. http://dx.doi.org/10.1100/tsw.2007.213

[40] Lee, M.J., Burau, K.D. and Dafny, N. (2013) Behavioral Daily Rhythmic Activity Pattern of Adolescent Female Rat Is Modulated by Acute and Chronic Cocaine. Journal of Neural Transmission, 120, 733-744. http://dx.doi.org/10.1007/s00702-012-0929-1

[41] Lee, M.J., Yang, P.B., Wilcox, V.T., Burau, K.D., Swann, A.C. and Dafny, N. (2009) Does Repetitive Ritalin Injection Produce Long-Term Effects on SD Female Adolescent Rats? Neuropharmacology, 57, 201-207. http://dx.doi.org/10.1016/j.neuropharm.2009.06.008

[42] Algahim, M.F., Yang, P.B., Burau, K.D., Swann, A.C. and Dafny, N. (2010) Repetitive Ritalin Treatment Modulates the Diurnal Activity Pattern of Young SD Male Rats. Central Nervous System Agents in Medicinal Chemistry, 10, 111.

[43] Russo, S.J., Dietz, D.M., Damitriu, D. Morrison, J.H., Malenka, R.C. and Nestler, E.J. (2010) The Addicted Synapse: Mechanism of Synaptic and Structural Plasticity in Nucleus Accumbens. Trends in Neurosciences, 33, 267-276. 